\title{
From turbulence to landscapes: Logarithmic mean profiles in bounded complex systems
}

\author{
Milad Hooshyar $\odot$ \\ Princeton Environmental Institute and Princeton Institute for International and Regional Studies, \\ Princeton University, Princeton, New Jersey 08544, USA \\ Sara Bonetti 1 (] \\ Institute for Sustainable Resources, University College London, London WC1H ONN, United Kingdom \\ and Department of Environmental Systems Science, ETH Zurich, Zurich 8092, Switzerland \\ Arvind Singh (1) \\ Department of Civil, Environmental, and Construction Engineering, University of Central Florida, Orlando, Florida 32816, USA \\ Efi Foufoula-Georgiou (1) \\ Department of Civil and Environmental Engineering and Department of Earth System Science, \\ University of California, Irvine, Irvine, California 92697, USA \\ Amilcare Porporato* \\ Princeton Environmental Institute and Department of Civil and Environmental Engineering, \\ Princeton University, Princeton, New Jersey 08544, USA
}

(Received 19 August 2019; revised 5 February 2020; accepted 12 August 2020; published 14 September 2020)

\begin{abstract}
We show that similarly to the logarithmic mean-velocity profile in wall-bounded turbulence, the landscape topography presents an intermediate region with a logarithmic mean-elevation profile. Such profiles are present in complex topographies with channel branching and fractal river networks resulting from model simulation, controlled laboratory experiments, and natural landscapes. Dimensional and self-similarity arguments are used to corroborate this finding. We also tested the presence of logarithmic profiles in discrete, minimalist models of networks obtained from optimality principles (optimal channel networks) and directed percolation. The emergence of self-similar scaling appears as a robust outcome in dynamically different, but spatially bounded, complex systems, as a dimensional consequence of length-scale independence.
\end{abstract}

DOI: 10.1103/PhysRevE.102.033107

\section{INTRODUCTION}

The striking channel and ridge patterns in the land surface emerge from nonlocal erosion and exhibit several well-known scaling laws [1]. The complex networks of channels and their self-similar statistical properties have common features with other branch-forming systems [2,3] and have become a key example of out-of-equilibrium systems in statistical physics [4-7]. The analogy between the landscape self-similarity and the scale invariance observed in turbulence has been exploited to analyze landscape morphology [8,9]. In particular, Bonetti et al. [10] emphasized the parallels between the channelization cascade and the hierarchical pattern formation toward finer scales observed in the nonequilibrium systems such as hydrodynamic turbulence [11-13]. Here this analogy between turbulence and landscape evolution is strengthened by the discovery of a logarithmic region in the mean-elevation profiles,

*Corresponding author: aporpora@ princeton.edu which resembles the logarithmic scaling of the mean streamwise velocity in the intermediate region of wall-bounded turbulent flow [11,14-18].

In wall-bounded turbulence, the constancy of the slope of the logarithmic profile, commonly denoted by $1 / \kappa$, where $\kappa$ is the von Karman's constant, has been verified in numerous experimental and numerical studies [18-21]. Similarly, here we establish the existence of a logarithmic region in mean-elevation profiles of landscape topography, using a combination of numerical simulations and analysis of data from laboratory experiments and real landscapes, as well as dimensional considerations. We show that such scaling also emerges in landscapes constructed from optimal channel networks $[5,6,22]$ as well as from directed percolation, which is commonly considered the universality class of local nonequilibrium absorbing processes [23,24]. For high erosion rates, we obtain mean-elevation profiles consisting of a linear part close to the fixed-elevation boundary, similar to the viscous sublayer in turbulence [25], followed by a logarithmic profile at the intermediate distance from both the boundary and the central part of the domain, similar to the inertial sublayer in turbulence $[11,26]$. 


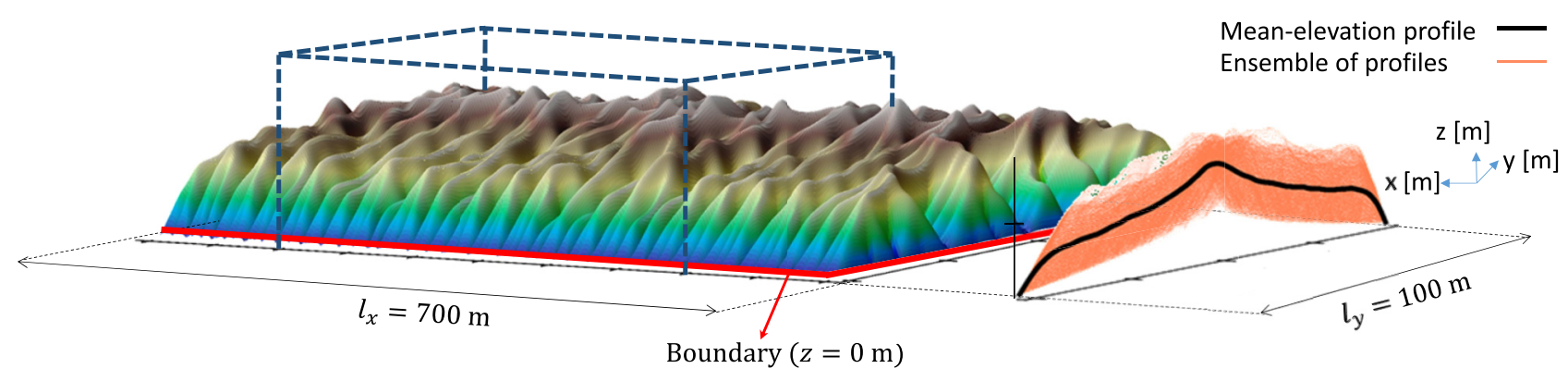

FIG. 1. An example of the steady-state surface obtained from the numerical solution of Eqs. (1) and (2). The simulation domain is $l_{x}=$ $700 \mathrm{~m}$ by $l_{y}=100 \mathrm{~m}$ and has $1-\mathrm{m}$ grid spacing. The boundary condition at the edges is constant elevation $z=0 \mathrm{~m}$. On the $y-z$ plane, the mean-elevation profile (black line) and the ensemble of profiles (red lines) are shown. To minimize the effect of domain size along the $x$ axis for the calculation of mean-elevation profile we used the surface within $100 \mathrm{~m} \leqslant x \leqslant 600 \mathrm{~m}$ as highlighted by the dashed box.

The logarithmic scaling is brought about by competition between the channel-forming mechanism and the geometric restriction of the boundary; the similarity with the competition between turbulence fluctuations and viscous damping at the wall hints at a common length-scale independence of complex systems with spatially organized patterns encroaching towards boundaries.

Before delving into the specific arguments of the paper, it should be pointed out that, similarly to the debate in nearwall turbulence regarding the logarithmic versus power-law profiles, here one could argue that the self-similar behavior of the mean-elevation profile might be the result of a more subtle type of self-similarity, entailing an intermediate asymptotic behavior leading to a power-law scaling (self-similarity of the second type [27]). We do not enter in this argument here, but only note that this does not undermine the main point of the presence of a common self-similar behavior among complex spatial systems (fractal river networks and the related landscapes, turbulence, and directed percolation) when their "branching patterns" interact with a boundary wall.

\section{LANDSCAPE EVOLUTION EQUATIONS}

We first consider the evolution of the land surface elevation in the detachment-limited condition [28-30], resulting from a balance between diffusive soil creep, fluvial erosion, and uplift:

$$
\frac{\partial z}{\partial t}=D \Delta z-K a^{m}|\nabla z|^{n}+U
$$

where $z(x, y, t)$ is the surface elevation at time $t$ and location $(x, y), D$ is the soil diffusivity, and $U$ is the tectonic uplift rate. The erosion term $K a^{m}|\nabla z|^{n}$, with parameters $K, m$, and $n$, is the main destabilizing term which generates the network of channels, similar to the nonlinear inertial term in high Reynolds number flow. The variable $a(x, y, t)$ is defined as the contributing area per unit contour-line length. The presence of $a$ in Eq. (1) follows from a condition of steady-state water depth generated by a unit rainfall rate under the assumption that water moves in the direction of the local slope with a constant velocity. As a result [10,31], $a$ is given by

$$
\nabla \cdot\left(a \frac{\nabla z}{|\nabla z|}\right)=-1
$$

By nondimensionalizing the system of Eqs. (1) and (2), Bonetti et al. [10] derived the dimensionless number $\mathcal{C}_{\mathcal{I}}$ which quantifies the relative impact of erosion to diffusive transport and uplift,

$$
\mathcal{C}_{\mathcal{I}}=\frac{K l^{m+n}}{D^{n} U^{1-n}}
$$

where $l$ is a typical length scale of the domain. Increasing $\mathcal{C}_{\mathcal{I}}$ results in a more dissected surface with branching channels [10], thus playing a role similar to that of the Reynolds number controlling the transition from laminar to turbulent flow.

Here, in analogy with a channel flow between parallel plates, we numerically solved the system of Eqs. (1) and (2) in a long strip of land, unbounded in the $x$ direction and with constant elevation at two sides, $z(x, 0)=z\left(x, l_{y}\right)=0 \mathrm{~m}$, where $l_{y}$ is the width in the direction $y$ (see Fig. 1). After reaching steady state, the mean-elevation profile $\bar{z}(y)$ was obtained by averaging the $z$ field along the $x$ axis for $100 \mathrm{~m} \leqslant x \leqslant 600 \mathrm{~m}$ (Fig. 1) to minimize the effect of $l_{x}$ on the mean behavior of the surface. An example of the mean-elevation profile (solid black line) and an example of the ensemble of profiles (red lines) are shown in the $y-z$ plane in Fig. 1.

\section{A. Dimensional analysis and self-similarity}

When averaged along the $x$ direction, the surface properties (e.g., elevation and slope) only depend on $y$. Therefore, the elevation field $z(x, y)$ can be decomposed into the sum of the mean elevation $\bar{z}$ and fluctuations $z^{\prime}$ around the mean, similarly to the Reynolds decomposition $[8,32]$,

$$
z(x, y)=\bar{z}(y)+z^{\prime},
$$

where $\bar{z}(y)=\lim _{l_{x} \rightarrow \infty} \frac{1}{l_{x}} \int_{0}^{l_{x}} z(x, y) d x$. The mean slope of steady-state surface is controlled by the parameters which describe the diffusive transport, fluvial erosion, and tectonic activity [ $D, K, U$, and $m$ in Eq. (1) with $n=1$ ], the distance $y$ from the boundary, and two length scales in the $y$ and $z$ directions $\left(l_{y}\right.$ and $\left.z_{*}\right)$ [33]:

$$
\frac{d \bar{z}}{d y}=f_{1}\left(y, l_{y}, z_{*}, D, K, U, m\right) .
$$


Choosing $y, D$, and $z_{*}$ as fundamental dimensionally independent variables, the $\Pi$ theorem yields

$$
\frac{y}{z_{*}} \frac{d \bar{z}}{d y}=f_{2}\left(\frac{y}{l_{y}}, \frac{K y^{m+1}}{D}, \frac{U y^{2}}{D z_{*}}, m\right),
$$

from which simple manipulation of the variables leads to

$$
(m+1) \eta \frac{d \varphi}{d \eta}=f_{3}\left(\eta, \mathcal{C}_{\mathcal{I}}, \zeta, m\right),
$$

where $\mathcal{C}_{\mathcal{I}}$ is given by Eq. (3) with $n=1$ and $l=l_{y}$, while $\varphi=\frac{\bar{z}}{z_{*}}$ is the mean elevation $\bar{z}$ normalized by a factor $z_{*}$ which describes the overall elevation of the profile. Here, we used $z_{*}=\bar{z}_{\max }$, where $\bar{z}_{\max }$ is the average elevation at the divide $\left(y=l_{y} / 2\right)$. The dimensionless value $\mathcal{C}_{\mathcal{I}}$ is a global quantity (independent of $y$ ) reflecting the relative impact of fluvial erosion to diffusive transport [10]. The quantity $\eta=\frac{K y^{m+1}}{D}$ is a local variable with a form similar to that of $\mathcal{C}_{\mathcal{I}}$ but capturing the local relative contribution of those two processes, while $\zeta=\frac{U l_{y}^{2}}{D z_{*}}$ describes the relative impact of tectonic uplift to diffusive transport.

In a system with relatively small diffusive transport and dominated by erosion and uplift, $\mathcal{C}_{\mathcal{I}}$ and $\zeta$ take high values. The same argument also applies to $\eta$ except for locations close to the boundary. Thus, when the variables $\eta, \mathcal{C}_{\mathcal{I}}$, and $\zeta$ reach such an asymptotic condition, one may assume complete self-similarity [33] according to which the function $f_{3}$ is independent of these quantities:

$$
\eta \frac{d \varphi}{d \eta}=\kappa(m)
$$

where $\kappa$ is only a function of $m$. Integrating Eq. (8) yields

$$
\varphi=\kappa(m) \ln \eta+C,
$$

where $C$ is independent of $\eta$ but may still depend on $m$, $\mathcal{C}_{\mathcal{I}}$, and $\zeta$. Equation (9) describes the logarithmic scaling of the mean-elevation profile with respect to $\eta$, similarly to the classic results of Millikan (1939) [15], to predict the logarithmic turbulent velocity profile. The emergence of such a logarithmic profile is expected in systems dominated by erosion and away enough from both the boundary and the center of symmetry (high $\mathcal{C}_{\mathcal{I}}, \zeta$, and $\eta$ ). Similar dimensional analysis for the general value of $n$ can be found in Appendix B.

It should be noted that an alternative assumption of incomplete self-similarity or self-similarity of the second kind [27], $f_{3} \propto \eta^{\alpha}$, would lead to a power-law mean-elevation profile $\varphi \propto \eta^{\alpha}$. As already said in the Introduction, we do not discuss this issue here, leaving it to further contributions.

\section{B. Numerical simulations}

We numerically solved Eqs. (1) and (2) to examine the mean-elevation profile under different conditions of diffusive transport and fluvial erosion. The simulation domain was a $700 \mathrm{~m}$ by $100 \mathrm{~m}$ rectangular grid with constant elevation $z=0 \mathrm{~m}$ at the boundary, as shown in Fig. 1. The choice of a long domain allowed us to neglect the effect of the domain dimension along the $x$ axis, mimicking the case of an infinite strip.
The numerical scheme used is a semi-implicit method that uses the $D_{\infty}$ flow-direction algorithm to efficiently calculate the contributing area at each pixel in the discretized domain and then it is divided by grid size to compute the specific catchment area $a[10,31,34]$. The spatial grid spacing was $1 \mathrm{~m}$ and the time step of discretization $\Delta t$ was selected small enough to avoid numerical instabilities. As the initial condition, we used a tent-shaped surface plus random noise in which the local minima were filled. We performed simulations with $1 \leqslant \mathcal{C}_{\mathcal{I}} \leqslant 2 \times 10^{5}, 0.1 \leqslant m \leqslant 1$, and $0.8 \leqslant n \leqslant$ 1.2 to cover the range of parameters reported in the literature $[35,36]$. For simplicity, we used $D=0.005 \mathrm{~m}^{2} / \mathrm{yr}$ and changed $K$ to achieve the desired $\mathcal{C}_{\mathcal{I}}$ for a given $m$ value.

The accuracy and robustness of the adopted numerical method are discussed in depth in Ref. [37]. These analyses include the comparison of the steady-state solution in the unchannelized regime $\left(\right.$ small $\mathcal{C}_{I}$ ) for which the analytic solution is available. Anand et al. [37] also show that the simulated surfaces capture the analytic prediction of $\mathcal{C}_{\mathcal{I}}$ at the transition from smooth to channelized surface that is derived [10]. They used the special case of $m=n=1$ and $\mathcal{C}_{\mathcal{I}} \rightarrow \infty$ to compare the temporal dynamics of the mean domain elevation form numerical simulation with the exact analytic prediction.

Increasing $\mathcal{C}_{\mathcal{I}}$ (i.e., increasing the relative magnitude of erosion compared to soil creep) results in a more dissected surface with branching channels (see Ref. [10]). As shown in Fig. 2, for $\mathcal{C}_{\mathcal{I}}=10$, the emerging surface is smooth with no channels because the diffusive transport is dominant and prevents the growth of instability and channel formation. Under these conditions, the mean elevation profile can be obtained analytically for $m=n=1$ in terms of a hypergeometric function [10]. As $\mathcal{C}_{\mathcal{I}}$ exceeds a critical value $\approx 37$ from linear stability analysis for $m=n=1$ ), parallel channels emerge and, with higher $\mathcal{C}_{\mathcal{I}}$, the surface becomes further dissected with the development of secondary branches [10]. Thus, the channelization index $\mathcal{C}_{\mathcal{I}}$ plays a role similar to that of the Reynolds number in fluid motion where the initiation and further progression of instabilities (transition from laminar to turbulent flow) are controlled by this quantity. The formation of branching channels impacts the mean-elevation profile, as shown in Figs. 2(e)-2(g). It is evident that, as $\mathcal{C}_{\mathcal{I}}$ increases, the profiles become more uniform, similar to the flattening of the mean-velocity profile with increasing Reynolds number in turbulence [38].

Figure 3(a) shows (half of) the mean-elevation profile for a range of $\mathcal{C}_{\mathcal{I}}$ using the dimensionless variables $\varphi$ and $\eta$ with $z_{*}=\bar{z}_{\max }$ in a semi-log space for $m=0.7$. The tendency of the mean-elevation profile toward a logarithmic scaling [linear segment in the semi-log space of Fig. 3(a)] clearly appears beyond a certain value of $\mathcal{C}_{\mathcal{I}}$.

We computed the characteristics of the logarithmic segment of the nondimensional mean-elevation profile by fitting a function to the profile within $0 \leqslant y \leqslant l_{y} / 2$. This includes a linear part for the segment close to the boundary and a logarithmic function for the intermediate segment. We also considered an additional power function for the segment close to the divide to cover the whole profile. We found the best fits by maximizing the summation of $R^{2}$ values of the fit to each segment. The variables for optimization are two thresholds for the dimensionless distance from the boundary $\eta$ which 


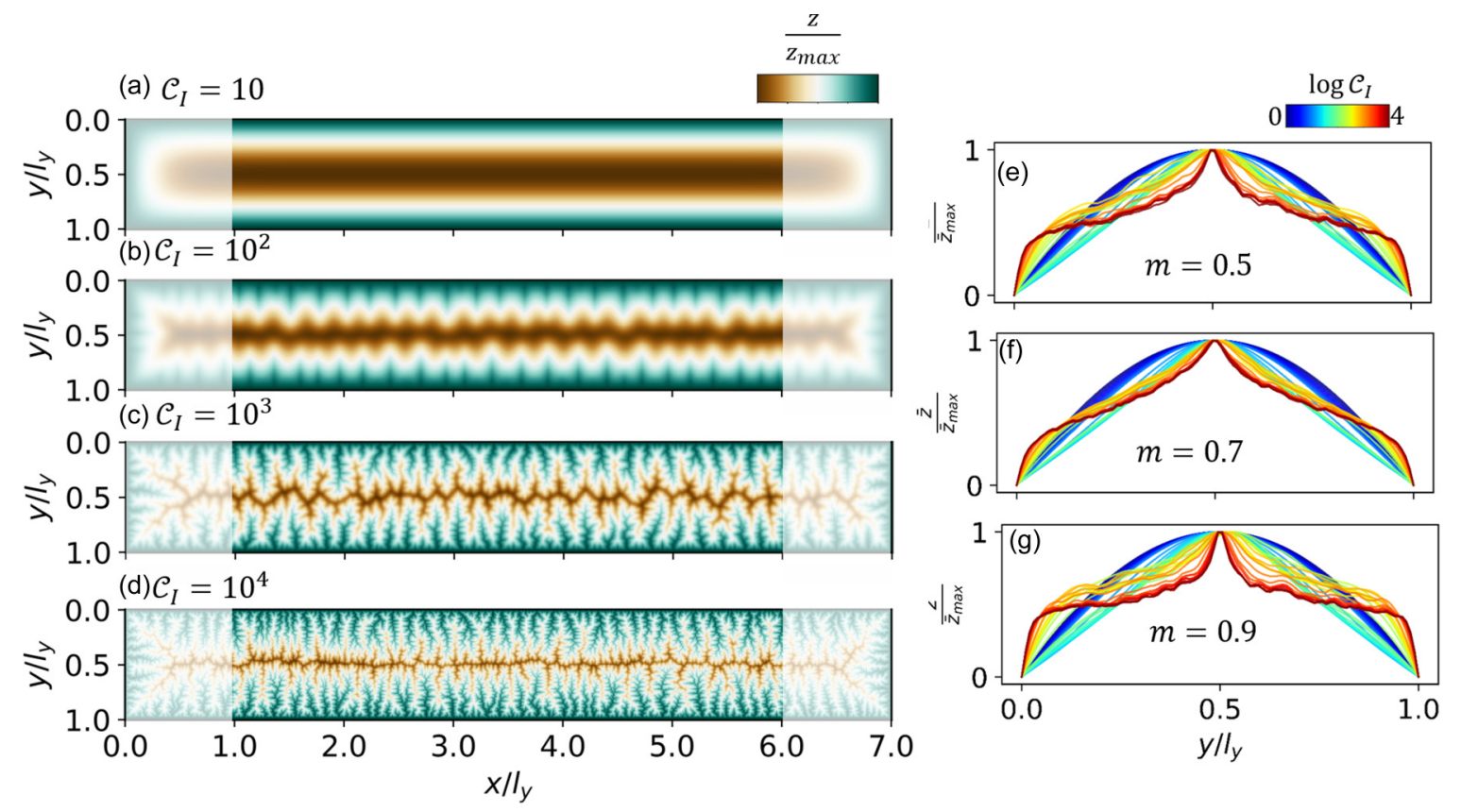

FIG. 2. The landscape surface and the mean-elevation profile for different $\mathcal{C}_{\mathcal{I}}$ and $m$. (a-d) The normalized elevation $z$ for $\mathcal{C}_{\mathcal{I}}=10,10^{2}$, $10^{3}$, and $10^{4}$ and $m=0.5$. The shaded segments at the boundaries are discarded for further extraction of mean-elevation profiles. (e-g) The average profile for a wide range of $\mathcal{C}_{\mathcal{I}}$ and $m=0.5, m=0.7$, and $m=0.9$. As $\mathcal{C}_{\mathcal{I}}$ increases (higher relative proportion of fluvial erosion to diffusive transport), the surface becomes more dissected with branching channels. This results in the evolution of the mean-elevation profile toward a flatter shape.

correspond to transition from the linear segment close to the boundary to the intermediate logarithmic segment and the transition from the logarithmic segment to the section close to the divide. Using such thresholds, simple linear regression gives the slope of the logarithmic profile denoted by $\kappa$. The logarithmic fits to the intermediate segments of the profiles for $\mathcal{C}_{\mathcal{I}}=2 \times 10^{5}$ are shown in Fig. 3(a). The slope of the logarithmic segment at high $\mathcal{C}_{\mathcal{I}}$ is only a function of $m$ as shown in Fig. 3(b).

\section{Laboratory and natural landscapes}

We also analyzed the data from a landscape evolution experiment performed using the Experimental Landscape Evolution (XLE) facility in the St. Anthony Falls Laboratory at the University of Minnesota, described in detail in
Refs. [39,40]. The parameters modulating fluvial erosion and diffusion needed to analyze the logarithmic scaling were estimated, assuming a steady-state governed by Eqs. (1) and (2) with $n=1$ as explained in Appendix A. Having $D, K$, and $m$ from the experimental surfaces, we computed the slope of the logarithmic profile $\kappa$ for the 16 surfaces with the same optimization algorithm used to analyze the surfaces from the numerical simulation. The surfaces from the physical experiment also display a logarithmic scaling [see Fig. 4(b)] with slopes $\kappa$ that lie close to the results from the numerical simulation [Fig. 3(b)].

To investigate the emergence of logarithmic meanelevation profiles in real landscapes, we analyzed highresolution topographic data from a basin located in the Calhoun Critical Zone in South Carolina, USA [Fig. 4(c)]. To make an analogy with the boundary condition used
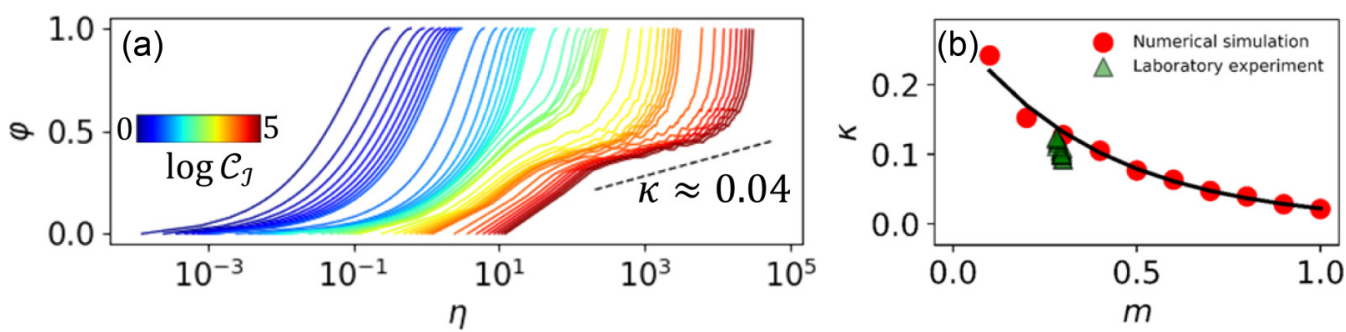

FIG. 3. The logarithmic profile in numerical simulations. (a) The dimensionless profiles corresponding to $0 \leqslant y \leqslant l_{y} / 2$ in a semilog space for $m=0.7$. The dimensionless quantities are defined as $\eta=\frac{K y^{m+1}}{D}$ and $\varphi=\frac{z}{z_{*}}$, with $z_{*}=\bar{z}_{\text {max }}$. Increasing $\mathcal{C}_{\mathcal{I}}$ leads to the emergence and further expansion of the logarithmic segment in the profiles. The dashed line is the fitted logarithmic line to the profile with $\mathcal{C}_{\mathcal{I}}=10^{5}$ and the slope is reported as $\kappa$. (b) The relationship between $\kappa$ and $m$. The slope $\kappa$ (average of simulations with $\mathcal{C}_{\mathcal{I}} \geqslant 10^{4}$ ) decreases monotonically with $m$, suggesting that higher values of $m$ result in flatter logarithmic profiles. The data points from a physical experiment are also shown and lie close to the results from the numerical simulation. 

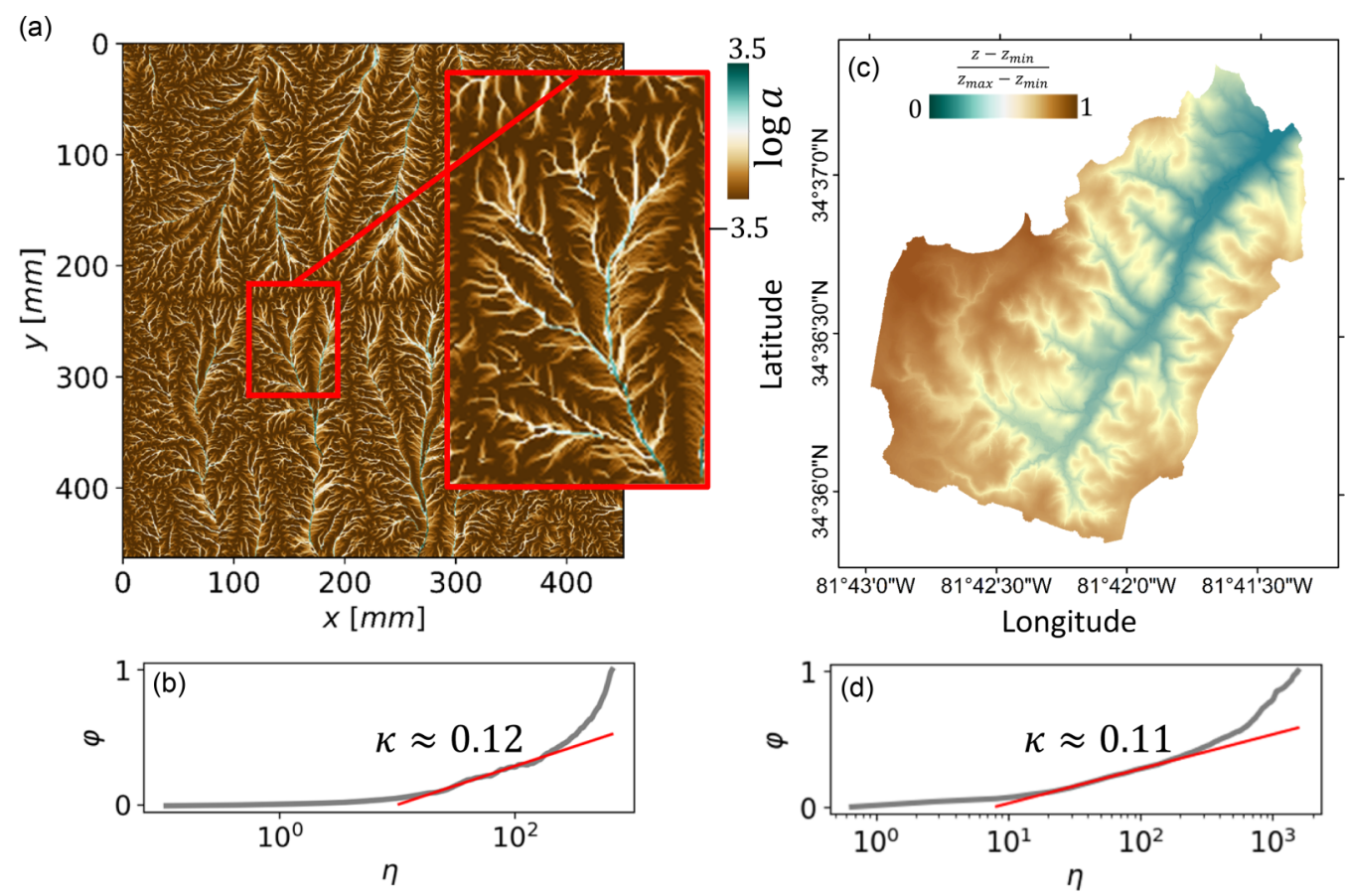

FIG. 4. The logarithmic profile in the experimental and natural landscapes. (a) The specific catchment area $a$ for an experimental landscape, and (b) its mean-elevation profile represented by the nondimensional quantities $\eta$ and $\varphi$. The fitted line to the logarithmic segment of the profile is shown by the red line with the slope reported as $\kappa \approx 0.12$. (c) The digital elevation model of a basin at the Calhoun Critical Zone in South Carolina, USA, and (d) its normalized mean-elevation profile which exhibits a logarithmic scaling with $\kappa \approx 0.11$.

in this work, we consider the main channel to be analogous to a fixed-elevation boundary and define $u(x, y)$ as the minimum distance from each location $(x, y)$ to the main channel. The mean-elevation profile is defined as $\bar{z}(d)=$ $\frac{1}{\left|S_{d}\right|} \sum_{(x, y) \in S_{d}} z(x, y)$, where $S_{d}$ is the set of points with $d \leqslant$ $u(x, y)<d+\Delta d$ and $\Delta d=5 \mathrm{~m}$. Figure 4(d) shows the normalized mean-elevation profile ( $\varphi$ versus $\eta$ ) for which parameters $D, K$, and $m$ were computed using a similar method explained in Appendix A for the physical experiment. The observed logarithmic scaling with the slope $\kappa \approx 0.11$ is consistent with the logarithmic scalings obtained from numerical simulations and laboratory experiments.

\section{ANALYSIS OF DISCRETE MINIMALIST MODELS}

To further corroborate the previous results and explore the emergence of a logarithmic profile in minimalist models of channelized landscapes, we also considered surfaces generated from two discrete minimalist models, namely optimal channel networks (OCNs) [41] and directed percolation (DP) [23].

\section{A. Optimal channel networks}

OCNs are configurations defined over a discrete domain and they locally minimize the total energy dissipation which is proportional to $\sum_{i \in I} A_{i}^{\gamma}$, where $\gamma$ is a constant, $I$ is the set of nodes in the domain, and $A_{i}$ is the drainage area of node $i[5,42]$. Such OCNs also describe landscapes with (locally) minimum average elevation and are directly related to the landscape evolution model formulation in the absence of diffusive transport [22]. Given the loopless configuration of an OCN, the corresponding elevation field can be constructed assuming a power relationship between slope $S_{i}$ and area $A_{i}$ at node $i$, i.e., $S_{i}=\beta A_{i}^{\gamma-1}$, where $\beta$ is a constant.

The optimality condition invokes a tendency toward aggregation of flow which in turn creates a branched network as shown in Fig. 5(a) for a $500 \mathrm{~m}$ by $100 \mathrm{~m}$ lattice with $1-\mathrm{m}$ spacing. The topology of the optimal configuration is controlled by the model parameter $\gamma$. The constructed surface elevation is directly followed from the optimal configuration as shown in Fig. 5(b) for the network of Fig. 5(a). Given the elevation field, the mean-elevation profile can be computed by averaging along the $x$ axis as shown in Fig. 5(c) in which a logarithmic scaling close the boundary is evident. The logarithmic scaling appears as a consequence of the competition between the branching invoked by optimality and the constant-elevation boundary condition, through the power-law relationship between slope and area.

\section{B. Directed percolation}

DP is widely considered to be the universality class of local nonequilibrium absorbing processes $[23,24]$ and has been found to describe the critical behavior of the laminar-turbulent transition [24,43-45].

In the DP framework, a set of nodes in a lattice can be either active or inactive with a probabilistic transition between these two states. Geometrically, DP describes the flux through a random medium in which channels (bonds) represent the connection between active nodes $[46,47]$ while the system has a preferred direction defined by external forcing. Here we used a modified form of DP to generate networks and their associated landscapes for a regular grid in a long rectangular 
(a)

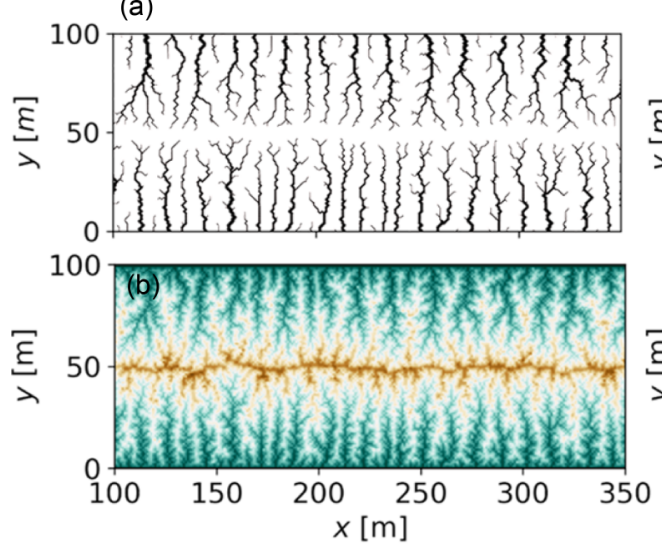

(d)
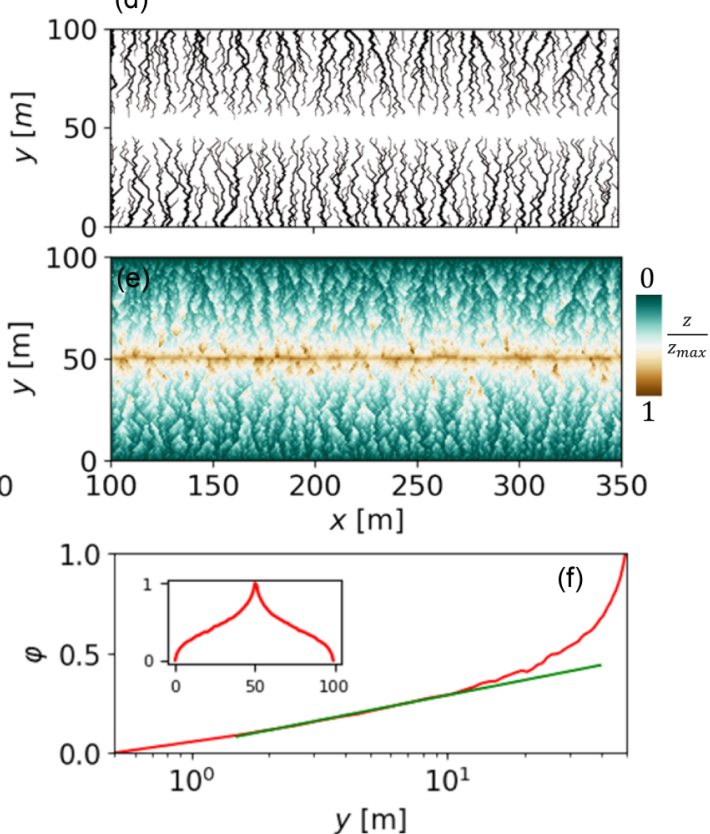

FIG. 5. The logarithmic profile in optimal channel networks (left) and directed percolation (right). Panels (a) and (b) show an optimal channel network (OCN) with $\gamma=0.5$ and a directed percolation (DP) network generated numerically in a $500 \mathrm{~m}$ by $100 \mathrm{~m}$ domain with $1-\mathrm{m}$ grid spacing. The lines represent the connection between nodes (links) and their width is proportional to the drainage area $A$ at their end. Panels (b) and (e) show the constructed surface elevations from the OCN and DP configurations. Panels (c) and (d) show the mean-elevation profiles computed for $100 \mathrm{~m}<x<400 \mathrm{~m}$ in linear (inset) and logarithmic horizontal axis for $0 \leqslant y \leqslant l_{y} / 2$. The existence of a logarithmic scaling is evident as marked by the fitted lines in green.

domain in which the preferred direction is imposed by gravity to ensure an overall flux toward the boundary.

For our analysis of landscapes and the analogy with turbulence (high $\mathcal{C}_{I}$ and high $\mathrm{Re}$ ), we focused on the asymptotic condition in which all nodes are active, although channels are generated randomly. Starting from the middle of the domain, the nodes are randomly connected to only one of three nodes located downstream towards the domain boundary. Limiting the outgoing bonds to only one assures the formation of antiarborescence trees with their roots at the domain boundary [see Fig. 5(d)].

As for the OCN, the elevation field was computed by setting zero elevation at boundary nodes and assuming a power relationship between slope and area [see Fig. 5(e)]. The meanelevation profile [Fig. 5(c)] was obtained by averaging the constructed elevation field along the $x$ axis. The logarithmic scaling in the mean-elevation profile is evident in Fig. 5(f) as highlighted by the fitted line. The network configuration from DP [Fig. 5(d)] is visually different (less branched) from that of OCN [Fig. 5(a)] due to apparently more randomness compared to the constraints imposed by optimality in OCN. Even in this case, however, the quenching of the branching pattern at the boundary of constant, lower elevation brings about an evident logarithmic profile, suggesting that DP may be a model of lesser complexity that can generate such a logarithmic mean-elevation profile.

\section{CONCLUSIONS}

Inspired by the resemblance between the progressive surface dissection and flattening of mean-elevation profile and the turbulence cascade and the mean velocity profile [32,38], we explored the existence of a self-similar, logarithmic region in mean-elevation profiles similar to the logarithmic scaling of streamwise velocity in wall-bounded turbulent flows $[11,18,26,48]$.

The fact that we found a logarithmic region in minimalist models of landscape evolution, laboratory experiments, and natural data hints at the generality of such scaling. Similar robustness to both boundary conditions and physical processes is present in turbulent velocity profiles, where a logarithmic region appears in both smooth and rough walls, as well as within different levels of approximation (e.g., direct numerical simulations and large-eddy simulation) $[17,49,50]$. The logarithmic scaling persists for a wide range of model parameters, in agreement with the robustness of the logarithmic scaling for different types of fluids (e.g., Newtonian and non-Newtonian) $[51,52]$.

It would be of interest to try to link the existence of the logarithmic scaling to the optimality principles (e.g., OCNs) and the related variational principles often advocated in landscape models $[1,4,5,41]$, as well as in turbulence in relation to viscous dissipation maximization [53,54]. Recently, Hooshyar et al. [22] showed that the landscape evolution model could be formulated as a variational problem of extremizing the average domain elevation.

In this paper, we did not enter into the debate about whether the observed self-similar scaling could actually be of the second type rather than the first type. While our results suggest the presence of logarithmic profiles, and therefore of first type similarity, it would certainly be of interest to perform more scrupulous scrutiny aimed at assessing this 
point. We leave this to future research, with only the comment that this will not undermine our main conclusion regarding the fact that landscapes and turbulence, both examples of complex systems out-of-equilibrium, exhibit a cascade mechanism [10,32] leading to a self-similar behavior in the mean profile where the progression of branching is hindered by boundaries.

While the dimensional analysis presented here does not "prove" any self-similar behavior [33], it helps explain the logarithmic scaling as the outcome of a self-similarity that is then validated in numerical simulations, natural landscapes, and laboratory experiments. The findings here build on the previous observations regarding the common behavior of landscapes and turbulence [8-10] for which logarithmic scaling follows from a similar line of reasoning [15]. It should be clear that this does not establish a common physical mechanism in these two systems, the answer to which requires proving that these two systems are from the same universality class and perhaps at different levels of complexity. It does however show that this self-similar scaling is a robust outcome in dynamically different complex systems, emerging as a dimensional consequence of length-scale independence, when they are spatially bounded.

\section{ACKNOWLEDGMENTS}

The comments of the anonymous reviewers are gratefully acknowledged. A.P. acknowledges support from the U.S. National Science Foundation (NSF) under Grants No. EAR1331846 and No. EAR-1338694 and from British Petroleum (BP) through the Carbon Mitigation Initiative (CMI) at Princeton University. M.H. acknowledges support from the Princeton Institute for International and Regional Studies (PIIRS) and the Princeton Environmental Institute (PEI). E.F. acknowledges support from the U.S. National Science Foundation (NSF) Grants No. DMS-1839336, No. EAR-1242458, and No. EAR-1811909.

\section{APPENDIX A: ANALYSIS OF THE LABORATORY EXPERIMENTS}

The parameters modulating fluvial erosion and diffusion needed to analyze the logarithmic scaling were estimated assuming a steady-state governed by Eqs. (1) and (2) with $n=1$ [36]. The specific catchment area $a$ was computed by dividing the total contributing area from $D_{\infty}$ by the grid spacing of the scanned surfaces $(0.5 \mathrm{~mm})$ [34]. Following Ref. [55] and focusing on the regions with small fluvial erosion (hilltops with small $a$ and $|\nabla z|$ ), from Eq. (1), $D$ was approximated as

$$
D=-\frac{U}{\triangle z_{a|\nabla z| \rightarrow 0}} \text {. }
$$
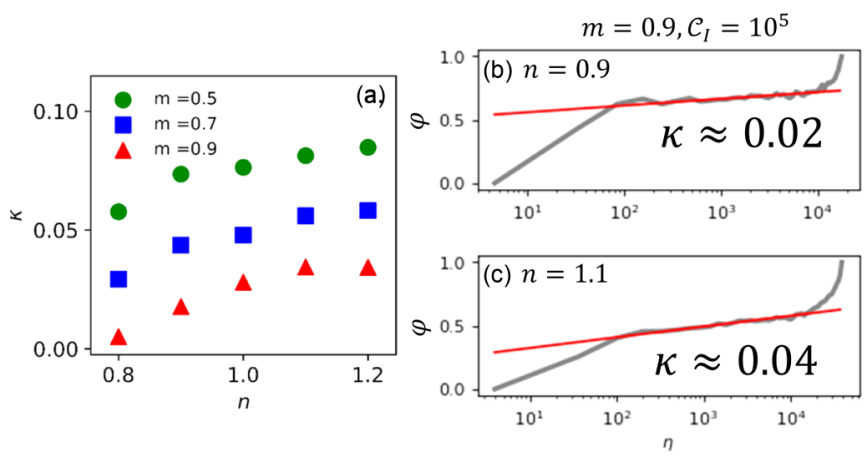

FIG. 6. The dependence of the slope of logarithmic scaling on the parameter $n$. (a) The logarithmic scaling persists for a range of $n$ and the slope $\kappa$ increases with higher $n$. (b, c) The nondimensionalized mean-elevation profiles for two cases with the same $m=0.9$ and $\mathcal{C}_{\mathcal{I}}=10^{5}$, but different $n$. In both cases the logarithmic scaling is easily detectable.

Given $D$ and from Eq. (A1) at steady state, $K$ and $m$ can be estimated by fitting a power function to $\frac{D \triangle z+U}{|\nabla z|}$ versus $a$ relationship [40,55]:

$$
\frac{D \triangle z+U}{|\nabla z|}=K a^{m} .
$$

Having $D, K$, and $m$ from the experimental surfaces, we computed the slope of the logarithmic profile $\kappa$ for the 16 surfaces with the same optimization algorithm used to analyze the surfaces from the numerical simulation.

\section{APPENDIX B: EFFECT OF $\boldsymbol{n}$ ON THE LOGARITHMIC SCALING}

The self-similarity arguments presented in the paper for $n=1$ can be readily generalized with respect to $n$ by defining $\eta=\frac{K y^{m+n}}{D^{n} U^{1-n}}$ and using $\mathcal{C}_{\mathcal{I}}$ from Eq. (3) with $l=l_{y}$. Following the same line of reasoning, one can find

$$
\varphi=\kappa(m, n) \ln \eta+C
$$

where $\kappa$ is a function of both $m$ and $n$. To reveal details on the functional dependence of $\kappa$ on $n$, we ran an additional 300 simulations with $\mathcal{C}_{\mathcal{I}} \geqslant 10^{4} ; m=0.5,0.7$, and $0.9 ; n=$ $0.8,0.9,1,1.1$, and 1.2; and the same boundary and initial conditions discussed earlier. Our results clearly show that the logarithmic scaling emerges for a range of parameter $n$ [Fig. 6(b) and 6(c)] and the slope $\kappa$ increases with higher $n$ values [Fig. 6(a)].
[1] I. Rodríguez-Iturbe and A. Rinaldo, Fractal River Basins: Chance and Self-Organization (Cambridge University, Cambridge, England, 2001).

[2] S. Kramer and M. Marder, Evolution of River Networks, Phys. Rev. Lett. 68, 205 (1992).

[3] A. Arneodo, F. Argoul, E. Bacry, J. F. Muzy, and M. Tabard, Golden Mean Arithmetic in the Fractal Branching of Diffusion-Limited Aggregates, Phys. Rev. Lett. 68, 3456 (1992).

[4] K. Sinclair and R. C. Ball, Mechanism for Global Optimization of River Networks from Local Erosion Rules, Phys. Rev. Lett. 76, 3360 (1996).

[5] A. Rinaldo, A. Maritan, F. Colaiori, A. Flammini, R. Rigon, I. Rodriguez-Iturbe, and J. R. Banavar, 
Thermodynamics of Fractal Networks, Phys. Rev. Lett. 76, 3364 (1996).

[6] J. R. Banavar, F. Colaiori, A. Flammini, A. Giacometti, A. Maritan, and A. Rinaldo, Sculpting of a Fractal River Basin, Phys. Rev. Lett. 78, 4522 (1997).

[7] J. R. Banavar, F. Colaiori, A. Flammini, A. Maritan, and A. Rinaldo, Scaling, optimality, and landscape evolution, J. Stat. Phys. 104, 1 (2001).

[8] C. Paola, Incoherent structure: Turbulence as a metaphor for stream braiding, in Coherent Flow Structures in Open Channels, edited by P. J. Ashworth, S. J. Bennett, J. L. Best, and S. J. McLelland (Wiley, New York, 1996), pp. 706-723.

[9] P. Passalacqua, F. Porté-Agel, E. Foufoula-Georgiou, and C. Paola, Application of dynamic subgrid-scale concepts from large-eddy simulation to modeling landscape evolution, Water Resour. Res. 42, W06D11 (2006).

[10] S. Bonetti, M. Hooshyar, C. Camporeale, and A. Porporato, Channelization cascade in landscape evolution, Proc. Natl. Acad. Sci. U.S.A. 117, 1375 (2020).

[11] H. Tennekes, J. L. Lumley, J. Lumley et al., A First Course in Turbulence (MIT, Cambridge, MA, 1972).

[12] G. R. Chavarria, C. Baudet, and S. Ciliberto, Hierarchy of the Energy Dissipation Moments in Fully Developed Turbulence, Phys. Rev. Lett. 74, 1986 (1995).

[13] A. Frishman and C. Herbert, Turbulence Statistics in a TwoDimensional Vortex Condensate, Phys. Rev. Lett. 120, 204505 (2018).

[14] L. Prandtl, 7. Bericht uber untersuchungen zur ausgebildeten turbulenz, ZAMM-J. Appl. Math. Mech./Z. Angew. Math. Mech. 5, 136 (1925).

[15] C. M. Millikan, A critical discussion of turbulent flows in channels and circular tubes, in Proceedings of the 5th International Congress of Applied Mechanics, Harvard University and MIT (Wiley Online Library, 1939).

[16] P. Bradshaw and G. P. Huang, The law of the wall in turbulent flow, Proc. R. Soc. London, Ser. A 451, 165 (1995).

[17] J. Jiménez, Cascades in wall-bounded turbulence, Annu. Rev. Fluid Mech. 44, 27 (2012).

[18] P. Luchini, Universality of the Turbulent Velocity Profile, Phys. Rev. Lett. 118, 224501 (2017).

[19] J. Nikuradse, Gesetzmäßigkeiten der turbulenten strömung in glatten rohren (nachtrag), Forsch. Ingenieurwes. 4, 44 (1933).

[20] J. G. M. Eggels, F. Unger, M. H. Weiss, J. Westerweel, R. J. Adrian, R. Friedrich, and F. T. M. Nieuwstadt, Fully developed turbulent pipe flow: A comparison between direct numerical simulation and experiment, J. Fluid Mech. 268, 175 (1994).

[21] S. C. C. Bailey, M. Vallikivi, M. Hultmark, and A. J. Smits, Estimating the value of von Kármán's constant in turbulent pipe flow, J. Fluid Mech. 749, 79 (2014).

[22] M. Hooshyar, S. K. Anand, and A. Porporato, Variational analysis of landscape elevation and drainage networks, Proc. R. Soc. A 476, 20190775 (2020).

[23] H.-K. Janssen, On the nonequilibrium phase transition in reaction-diffusion systems with an absorbing stationary state, Z. Phys. B: Condens. Matter 42, 151 (1981).

[24] N. Goldenfeld and H.-Y. Shih, Turbulence as a problem in non-equilibrium statistical mechanics, J. Stat. Phys. 167, 575 (2017).
[25] B. J. Mckeon, J.-d. Li, W. Jiang, J. F. Morrison, and A. J. Smits, Further observations on the mean velocity distribution in fully developed pipe flow, J. Fluid Mech. 501, 135 (2004).

[26] I. Marusic, J. P. Monty, M. Hultmark, and A. J. Smits, On the logarithmic region in wall turbulence, J. Fluid Mech. 716, R3 (2013).

[27] G. Barenblatt and N. Goldenfeld, Does fully developed turbulence exist? Reynolds number independence versus asymptotic covariance, Phys. Fluids 7, 3078 (1995).

[28] A. D. Howard, A detachment-limited model of drainage basin evolution, Water Resour. Res. 30, 2261 (1994).

[29] N. Izumi and G. Parker, Inception of channelization and drainage basin formation: Upstream-driven theory, J. Fluid Mech. 283, 341 (1995).

[30] M. Hooshyar, A. Singh, and D. Wang, Interbasin and intrabasin competitions control drainage network density, Geophys. Res. Lett. 46, 661 (2019).

[31] S. Bonetti, A. Bragg, and A. Porporato, On the theory of drainage area for regular and non-regular points, Proc. R. Soc. A: Math. Phys. Eng. Sci. 474, 20170693 (2018).

[32] O. Reynolds, IV. On the dynamical theory of incompressible viscous fluids and the determination of the criterion, Philos. Trans. R. Soc. London, Ser. A 186, 123 (1895).

[33] G. I. Barenblatt, Scaling, Self-Similarity, and Intermediate Asymptotics: Dimensional Analysis and Intermediate Asymptotics (Cambridge University, Cambridge, England, 1996).

[34] D. G. Tarboton, A new method for the determination of flow directions and upslope areas in grid digital elevation models, Water Resour. Res. 33, 309 (1997).

[35] J. T. Perron, W. E. Dietrich, and J. W. Kirchner, Controls on the spacing of first-order valleys, J. Geophys. Res.: Earth Surf. 113, F04016 (2008).

[36] K. Sweeney, J. Roering, and C. Ellis, Experimental evidence for hillslope control of landscape scale, Science 349, 51 (2015).

[37] S. K. Anand, M. Hooshyar, and A. Porporato, Linear layout of multiple flow-direction networks for landscape-evolution simulations, Envir. Modell. Software, 104804 (2020).

[38] P. K. Kundu, I. M. Cohen, and D. W. Dowling, Fluid Mechanics, 5th ed. (Elsevier, Amsterdam, 2011).

[39] A. Singh, L. Reinhardt, and E. Foufoula-Georgiou, Landscape reorganization under changing climatic forcing: Results from an experimental landscape, Water Resour. Res. 51, 4320 (2015).

[40] M. Hooshyar, A. Singh, D. Wang, and E. Foufoula-Georgiou, Climatic controls on landscape dissection and network structure in the absence of vegetation, Geophys. Res. Lett. 46, 3216 (2019).

[41] R. Rigon, A. Rinaldo, I. Rodriguez-Iturbe, R. L. Bras, and E. Ijjasz-Vasquez, Optimal channel networks: A framework for the study of river basin morphology, Water Resour. Res. 29, 1635 (1993).

[42] A. Rinaldo, R. Rigon, J. R. Banavar, A. Maritan, and I. Rodriguez-Iturbe, Evolution and selection of river networks: Statics, dynamics, and complexity, Proc. Natl. Acad. Sci. U.S.A. 111, 2417 (2014).

[43] Y. Pomeau, Front motion, metastability and subcritical bifurcations in hydrodynamics, Phys. D (Amsterdam, Neth.) 23, 3 (1986). 
[44] H.-Y. Shih, T.-L. Hsieh, and N. Goldenfeld, Ecological collapse and the emergence of traveling waves at the onset of shear turbulence, Nat. Phys. 12, 245 (2016).

[45] M. Sipos and N. Goldenfeld, Directed percolation describes lifetime and growth of turbulent puffs and slugs, Phys. Rev. E 84, 035304(R) (2011).

[46] S. R. Broadbent and J. M. Hammersley, Percolation processes: I. Crystals and mazes, in Mathematical Proceedings of the Cambridge Philosophical Society, Vol. 53 (Cambridge University, Cambridge, England, 1957), pp. 629-641.

[47] H. Hinrichsen, Non-equilibrium critical phenomena and phase transitions into absorbing states, Adv. Phys. 49, 815 (2000).

[48] T. Banerjee and G. Katul, Logarithmic scaling in the longitudinal velocity variance explained by a spectral budget, Phys. Fluids 25, 125106 (2013).

[49] J. Kim, P. Moin, and R. Moser, Turbulence statistics in fully developed channel flow at low Reynolds number, J. Fluid Mech. 177, 133 (1987).
[50] W. Cheng and R. Samtaney, Power-law versus log-law in wallbounded turbulence: A large-eddy simulation perspective, Phys. Fluids 26, 011703 (2014).

[51] K. C. Wilson and A. D. Thomas, A new analysis of the turbulent flow of non-Newtonian fluids, Canadian J. Chem. Eng. 63, 539 (1985).

[52] M. Rudman and H. M. Blackburn, Direct numerical simulation of turbulent non-Newtonian flow using a spectral element method, Appl. Math. Modell. 30, 1229 (2006).

[53] W. Malkus and L. Smith, Upper bounds on functions of the dissipation rate in turbulent shear flow, J. Fluid Mech. 208, 479 (1989).

[54] J. Bertram, Maximum kinetic energy dissipation and the stability of turbulent Poiseuille flow, J. Fluid Mech. 767, 342 (2015).

[55] J. T. Perron, J. W. Kirchner, and W. E. Dietrich, Formation of evenly spaced ridges and valleys, Nature (London) 460, 502 (2009). 